\title{
Prolonged analgesic duration of brachial plexus block by addition of dexamethasone to dexmedetomidine for sedation
}

\author{
Byungmuk Kim, Boohwi Hong, Sangwon Yun, Youngkwon Ko
}

Department of Anesthesiology and Pain Medicine , Chungnam national university hospital, Daejeon, Republic of Korea

\section{BACKGROUND and AIMS}

We previously reported that dexmedetomidine for sedation prolonged analgesic duration of brachial plexus block (BPB). However, the prolonged effect was limited only on the day of surgery and this led to severe pain during the night and sleep disturbance.

We hypothesized that the co-administration of dexamethasone with dexmedetomidine for sedation could significantly prolong the analgesic duration of BPB.

\section{METHODS}

Sixty patients received ultrasound guided supraclavicular BPB and randomly assigned to one of three groups; C group (Control, midazolam), DM group (Dexmedetomidine), DMDX group (Dexmedetomidine + Dexamethasone $10 \mathrm{mg}$ ).

Primary outcome was ratio of patients whose analgesic duration extended to 20 hours and time to first request for analgesia by survival analysis. Quality of sleep and pain score were also measured as secondary outcomes.

\begin{tabular}{lccc}
\hline \multicolumn{1}{c}{ GROUP } & $\begin{array}{c}\mathrm{C} \\
(\mathrm{N}=20)\end{array}$ & $\begin{array}{c}\mathrm{DM} \\
(\mathrm{N}=20)\end{array}$ & $\begin{array}{c}\text { DMDX } \\
(\mathrm{N}=20)\end{array}$ \\
\hline Sex $(\mathrm{F} / \mathrm{M})$ & $14 / 6$ & $8 / 12$ & $12 / 8$ \\
Age $(\mathrm{yr})$ & 57 & 48.5 & 59 \\
& {$[26.5 ; 66.0]$} & {$[36.5 ; 57.5]$} & {$[43.5 ; 68.5]$} \\
Heights $(\mathrm{cm})$ & 162.5 & 163.6 & 157 \\
& {$[155.4 ; 169.6]$} & {$[157.8 ; 172.1]$} & {$[151.2 ; 167.5]$} \\
Weight $(\mathrm{kg})$ & 63.5 & 62.6 & 55.1 \\
Surgery Time & {$[54.5 ; 71.1]$} & {$[55.0 ; 69.6]$} & {$[50.0 ; 71.5]$} \\
(min) & 69 & 60.5 & 63 \\
\hline
\end{tabular}

\section{RESULTS}

There was no case that the effective analgesic duration was over 20 hours in C and DM groups. Only 12 patients $(60.0 \%)$ were in DMDX group.

Time to first request for analgesia (median [interquatile range]) was significantly longer in DMDX group (24.0 [16.5, -] hours) than in DM and $C$ group $(10.5[9.0,14.0]$ and $7.0[7.0,8.0]$ hours) $(P<0.001)$.

Even though the rate of sleep disturbance on POD 0 was insignificant among 3 groups, DMDX group showed the better score of sleep quality than other 2 groups.

Postoperative maximum NRS pain score in DMDX group was significantly lower than in other two groups.

\begin{tabular}{|c|c|c|c|c|}
\hline GROUP & $\begin{array}{c}C \\
(N=20)\end{array}$ & $\begin{array}{c}\text { DM } \\
(\mathbf{N}=\mathbf{2 0})\end{array}$ & $\begin{array}{l}\text { DMDX } \\
(\mathrm{N}=20)\end{array}$ & p \\
\hline $\begin{array}{l}\text { Dexmedetomidine dose } \\
(\mu \mathrm{g})\end{array}$ & - & $\begin{array}{c}65.2 \\
{[58.0 ; 86.8]}\end{array}$ & $\begin{array}{c}59.0 \\
{[50.4 ; 72.8]}\end{array}$ & 0.172 \\
\hline $\begin{array}{l}\text { Number of patients with } \\
\text { extended analgesic dura- } \\
\text { tion of more than } 20 \text { hours }\end{array}$ & $0(0.0 \%)$ & $0(0.0 \%)$ & $12(60.0 \%)$ & $\stackrel{<}{<}$ \\
\hline $\begin{array}{l}\text { NRS pain Score at first } \\
\text { request for analgesic } \\
\text { POD 0 Sleep quality }\end{array}$ & $\begin{array}{c}4.0 \\
{[3.0 ; 5.0]}\end{array}$ & $\begin{array}{c}4.5 \\
{[3.0 ; 5.0]}\end{array}$ & $\begin{array}{c}2.0 \\
{[0.0 ; 4.0]}\end{array}$ & 0.015 \\
\hline Disturbance (yes) & $13(65.0 \%)$ & $10(50.0 \%)$ & $6(30.0 \%)$ & 0.085 \\
\hline $\begin{array}{l}\text { Satisfaction score } \\
(5 \text { point likert scale) }\end{array}$ & $\begin{array}{c}4.0 \\
{[3.5 ; 4.0]}\end{array}$ & $\begin{array}{c}4.5 \\
{[3.0 ; 5.0]}\end{array}$ & $\begin{array}{c}5.0 \\
{[4.0 ; 5.0]}\end{array}$ & 0.014 \\
\hline \multicolumn{5}{|l|}{ Maximum NRS pain Score } \\
\hline POD 0 & $\begin{array}{c}5.0 \\
{[5.0 ; 6.0]}\end{array}$ & $\begin{array}{c}5.0 \\
{[3.0 ; 5.0]}\end{array}$ & $\begin{array}{c}0.0 \\
{[0.0 ; 4.0]}\end{array}$ & $\begin{array}{c}< \\
0.001\end{array}$ \\
\hline POD 1 & $\begin{array}{c}3.0 \\
{[3.0 ; 4.0]}\end{array}$ & $\begin{array}{c}3.0 \\
{[2.0 ; 5.0]}\end{array}$ & $\begin{array}{c}2.0 \\
{[1.0 ; 2.5]}\end{array}$ & $\begin{array}{c}< \\
0.001\end{array}$ \\
\hline POD 2 & $\begin{array}{c}2.0 \\
{[2.0 ; 3.0]}\end{array}$ & $\begin{array}{c}2.0 \\
{[1.0 ; 3.0]}\end{array}$ & $\begin{array}{c}1.5 \\
{[0.5 ; 2.0]}\end{array}$ & 0.035 \\
\hline
\end{tabular}

Blood glucose

concentrations $(\mathrm{mg} / \mathrm{dl})$

Preoperative

$101 \quad 103$

113.0

[95.0;121.0] [98.0;108.5] [101.5;138.0] 0.208

$111 \quad 103 \quad 125.0$ $[105.0 ; 129.0][88.5 ; 115.5][105.5 ; 143.0] 0.105$

Postoperative Strata + control + DM + DMDX

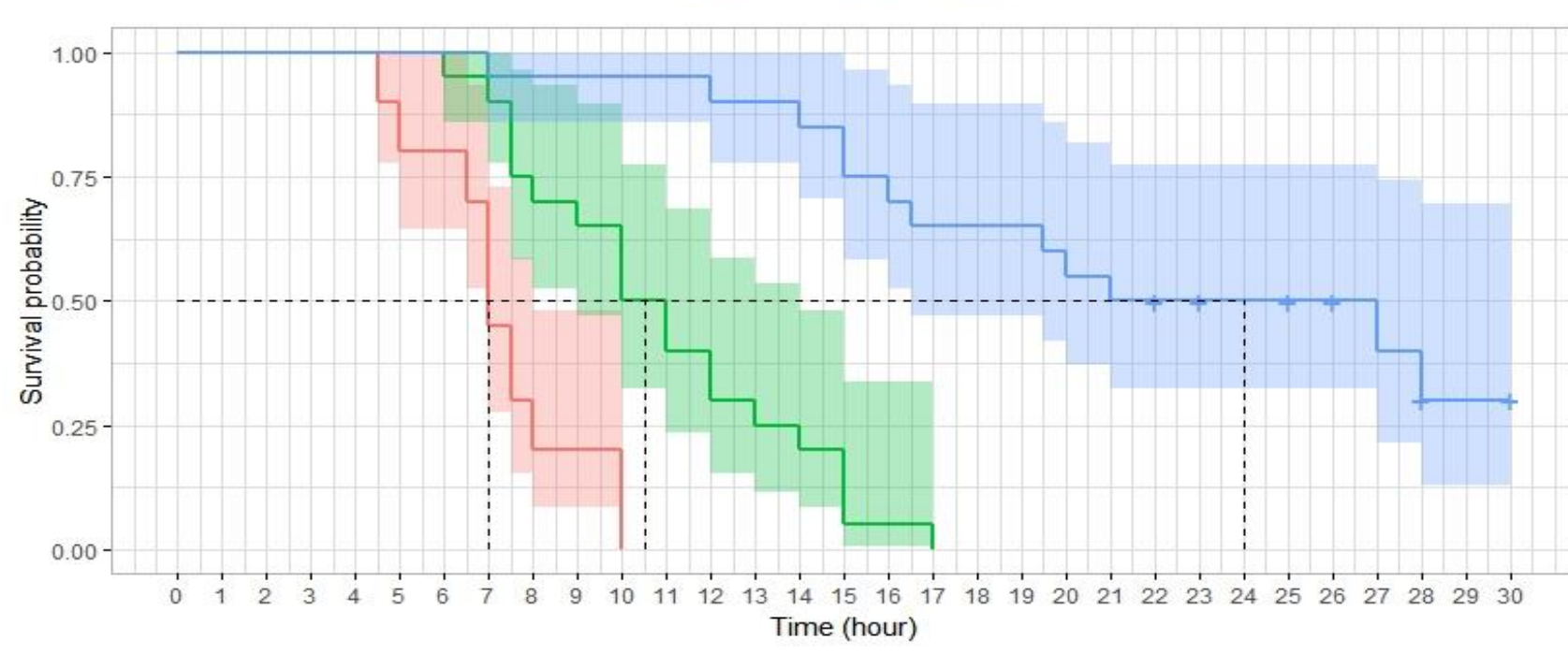

Number at risk

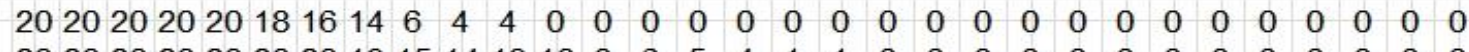

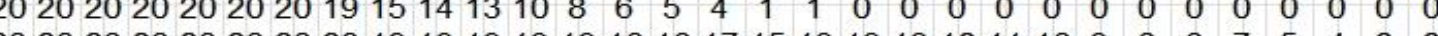
20202020202020201900

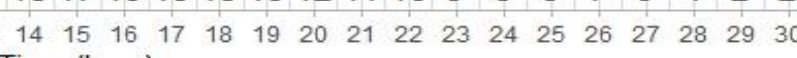

\section{CONCLUSIONS}

The addition of dexamethasone to dexmedetomidine for sedation in BPB seems to increase analgesic duration significantly and provide a favorable sleep quality to patients.

\section{REFERENCES}

1. Abdallah FW, Dwyer T, Chan VW. IV and perineural dexmedetomidine similarly prolong the duration of analgesia after interscalene brachial plexus block. Anesth. 2016;124(3):683-95.

2. B. Hong, W. Chung, Y, Ko. Sedation with dexmedetomidine prolongs the analgesic duration of brachial plexus block.

Anaesth Crit Care Pain Med 38 (2019) 231-236 